\title{
A Stochastic Approach in Determining Fire Size and Classification in Fire Risk Analysis and Monitoring
}

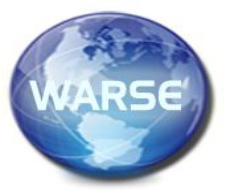

\author{
Rostam Salleh $^{1}$ \\ ${ }^{1}$ Industrial Automation Section \\ Universiti Kuala Lumpur Malaysia France Institute \\ 43560 Bandar Baru Bangi. \\ ${ }^{2}$ Sallehudin M.Haris / ${ }^{3}$ Rizauddin Ramli \\ Department of Mechanical and Materials Engineering \\ Faculty of Engineering \& Built Environment \\ Universiti Kebangsaan Malaysia \\ 43600 Bangi, Selangor Darul Ehsan, Malaysia. \\ sallehuddinmh@ukm.edu.my / rizauddin@ukm.edu.my
}

\begin{abstract}
This paper proposes to use the stochastic approach in evaluating the risk of fire incidents. The proposed methodology aims to incorporate the effect of uncertainty into the analysis which might produce a better overall assessment of the risk of fires. To estimate the probability of the risk of fire incidents, the following stochastic variables are used: humidity $(\mathrm{H})$, lux or light $(\mathrm{L})$, temperature $(\mathrm{T})$, and pressure (P). These variables are then represented with probability distribution curves. In this analysis, different curves for frequency of release, different fire diameter sizes and different leak sizes are also used. To obtain the fire risk as a probability distribution, a Monte Carlo simulation using Minitab analysis was performed. Finally, in order to estimate the probability of satisfying the risk tolerance criterion, the fire risk distribution curve obtained via the Monte Carlo simulation.
\end{abstract}

Key words: Fire risk; Uncertainty; Reliability.

\section{INTRODUCTION}

The Director-General of the Malaysian Fire and Rescue Department (FRD) has been reported to have said that fires throughout Malaysia in the year 2015 have caused losses amounting to an assessed value of RM 4.4 billion. According to him, the number of deaths increased by 10 percent, from 139 in 2014 to 153 cases in 2015. Johor recorded the highest number of casualties and damage caused by fires involving 11 deaths, 50 injuries, and an estimated loss of RM 925, 221.25. These statistics reveal that much more needs to be done to reduce the incidence of fire, which in turn will reduce and prevent the loss of life and property.
Structural fire analysis has been highly reliant on prescriptive rules in traditional codes. Despite their relatively simple implementation, these codes are rigid and they usually lead to expensive designs. To address the effects of uncertainty in engineering designs, a probabilistic approach has been provided as a rational framework, and this has been gradually incorporated in performance-based design guidelines [1]. In the quantitative structure-fire risk assessment of compartments, studies have been devoted to the development of stochastic models, experimental in investigations and statistical characterization of parameter, the model focuses on uncertainly of coefficients associated with burning rate, wall heat transfer processes and predicts the fire growth in a compartment for fire risk analysis[6]. Brandyberry and Apostolakis studied the ignition risk of consumer product in a building based on heat transfer mechanisms[3]. The study accounted for uncertainties in the ignition source (e.g.,surface area, amount of radiated heat) and in the target scenario (e.g.,density heat). The probability of ignition of a target object for a given exposure was obtained using a direct Monte Carlo method[4].

\section{EXPERIMENT METHOD}

The physical quantities under observation are given as:

A. Light: Light is an electromagnetic radiation which is visible to human eye. The light incidence over a surface is measured by luminance. Common Units: Lux (Lumen/m2), Candela (Lumen/Solid Angle)

B. Voltage: The electric potential difference between two points is called electric potential difference. Common Units: Volts (V), Joule/Coulomb. 
C. Temperature: It is a measure of Heat radiations. Common Units: Fahrenheit (F), Degree Celsius (o)C), Kelvin (K)

D. Relative Humidity: The humidity is measured by three ways as Absolute, Relative and Specific humidity.

E. Acceleration: The rate of change of velocity with respect to time of an object is called acceleration. Common Unit: meter/ $\sec 2$.

Crossbow Wireless Sensor Nodes in Figure 1 (popularly known as MICAz motes) with Gateway and Local Monitoring Server was deployed on a table inside the lab Figure 2. The amount of water vapor in the free air is then measured. Relative humidity is mostly used in many practical applications such as weather forecasting[18]. Common Units:[14]The relative humidity is usually expressed by percentage $\%$.

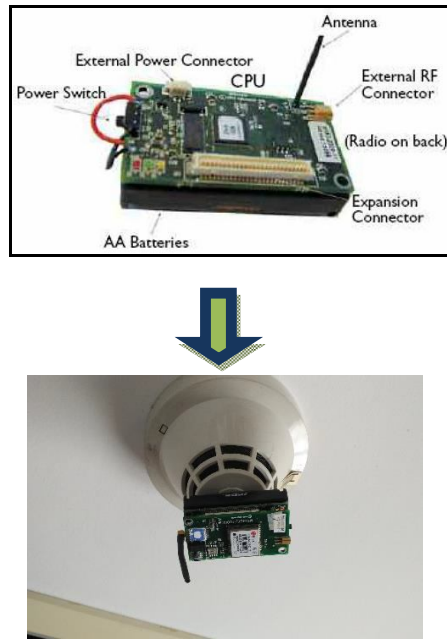

Figure 1 : WSN Meca2 Sensor

From the experiment methods, wireless sensor networks can be deployed for online monitoring of Voltage, Humidity, Temperature, Pressure, Light, and Acceleration inside a building as shown in Figure 4. The Crossbow MICAz motes are equipped with temperature, light, humidity, acceleration and pressure measurement sensors operating at $2.4 \mathrm{GHz}$ frequency[15]. These sensor node s are supplied with a 3 volts battery supply (two 1.5 volts, $500 \mathrm{mAh}$, pencil size cells, AA ratings)[5].

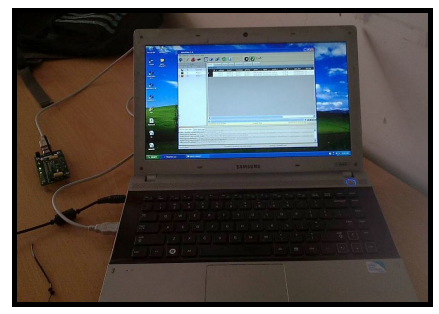

Figure 2 : WSN Meca2 Sensor Gateway

\begin{tabular}{|c|c|c|c|}
\hline Conditions & \multicolumn{3}{|c|}{ Air-condition on/off, Light on/off, Daytime/Night- } \\
time
\end{tabular}

Figure 3 : Stage of Fire based on number of candle

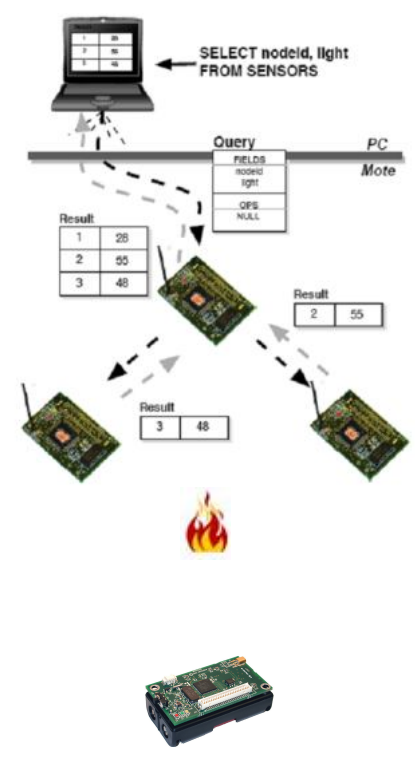

Figure 4 : Data Collection WSN Meca2 Sensor

Generally we cannot distinguish changes in the environment on the size of 1 to 2 lit candles in terms of brightness, temperature, and voltage humidity Figure 3 . By using sensor techniques and Meca2 UVtron sensor can detect fire in places and there can measure the size of the fire-giving to the risk of fire[19]. Uvtron sensors used to detect the existence of fire even with a small splash[6]. Meca2 sensor is able to measure the four variables in the environment, such as temperature, humidity, brightness, humidity temperature, pressure and voltage. The data obtained are collected for testing. Testing data made using statistical stochastics methods[7].

\section{RESULTS AND DISCUSSION}

The experiment used of several methods to distinguish the size of fire measuring the variables such as temperature, light(lux), humidity and pressure bar. All data are processed using stochastic data analysis method of statistic technique[8]. The difference for of the distribution curve be able to distinguish for the category type and size of the fire. Some categories are divided into surrounding during the no fire (L0), small candle flame (L1) and (L2) fire within 1.2 meter. 
Rostam Salleh et al., International Journal of Advanced Trends in Computer Science and Engineering, 8(1.6), 2019, 514 - 518

There are four (4) variable data retrieved through the readings such as Sensor Temperature (T), Light $(\mathrm{L})$, Humidity $(\mathrm{H})$, and Pressure (P). The experiments yielded four (4) different data distribution. However, the Humidity $(\mathrm{H})$ data reading provides the most significant value because it can distinguish the graph curve in three different circumstances. This distribution data can also find the value of the probability density function (PDF) and the cumulative density function (CDF)[9]. Indirectly, the given parameters can build a method to distinguish the parameter values of three fire types based on the intensity level of the fire. Figure 5 shows the graph Density curve for humidity PDF and CDF. From the graph of distribution, it is possible to distinguish the size of fire in accordance with L0, L1 and L2. The CDF curve in Figure 3 clearly determines the important range to evaluate the differences in the sizes of the fire [10]. Thus, it is easier to develop a more accurate algorithm for different fire sizes [11].

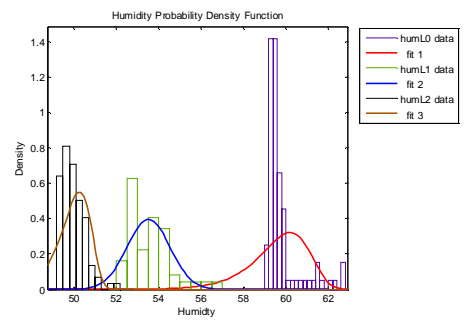

Figure 5: Humidity Probability Density Function

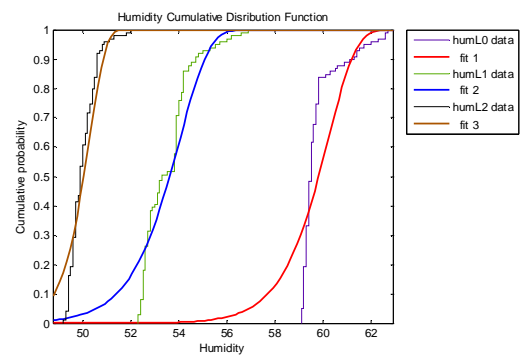

Figure 6: Humidity Cumulative Distribution Function

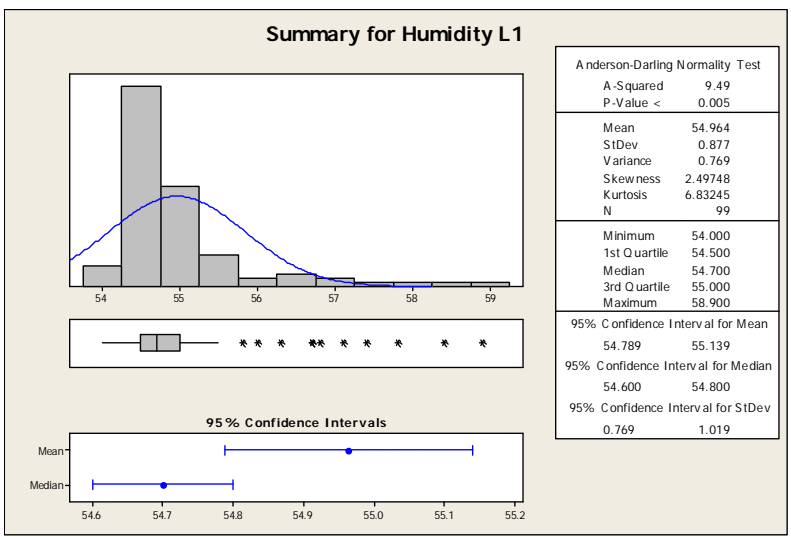

Figure 7 : Summary of Humidity L1
Table 1: A List of random variable $\mathrm{N}=99$ Humidity in fire models

A List of random variable $N=99$ Humidity in fire size models

\begin{tabular}{lllllll} 
Variable & Distribution & Mean & Std Deviation & Kurtosis & Skrew ness & Units \\
\hline Humd L0 & Normal & 0.2007 & 0.2302 & -2.004 & 0.263 & $\mathrm{c}$ \\
Humd L1 & Normal & 54.964 & 0.877 & 6.832 & 2.497 & $\mathrm{c}$ \\
Humd L2 & Normal & 69.278 & 1.733 & 3.567 & 2.027 & $\mathrm{c}$ \\
\multirow{2}{*}{ Humd L3 } & Normal & 71.233 & 1.823 & 3.888 & 2.018 & $\mathrm{c}$ \\
\hline
\end{tabular}

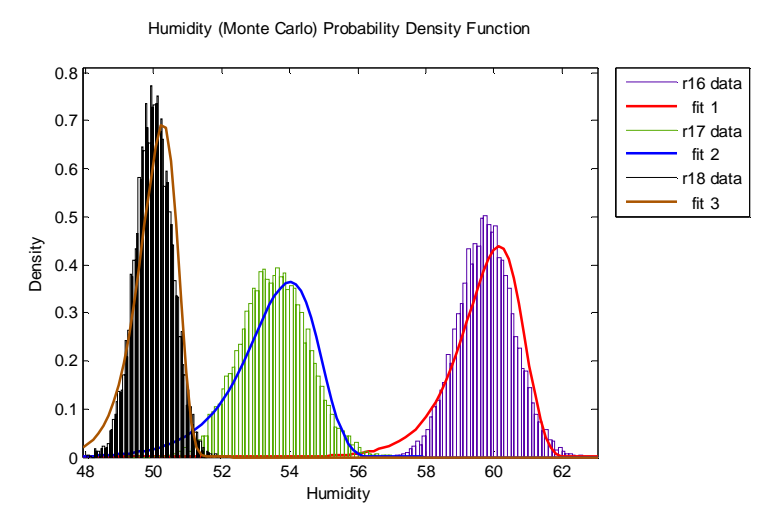

Figure 8: Monte Carlo Probability Density Function

Based on experimental fire data was taken only within a few minutes and data samples can be obtained 100 times. The candles burn factor could not be measured in the time period. Similarly, the factors caused the invoice distribution around as wind and so on. The Monte Carlo method is used to add data such as sampling was increased to up 10,000 times[12]. The reading shows a more accurate output with strengthen and support again to find the true value. Monte Carlo Probability Function PDF shown in Figure 8.

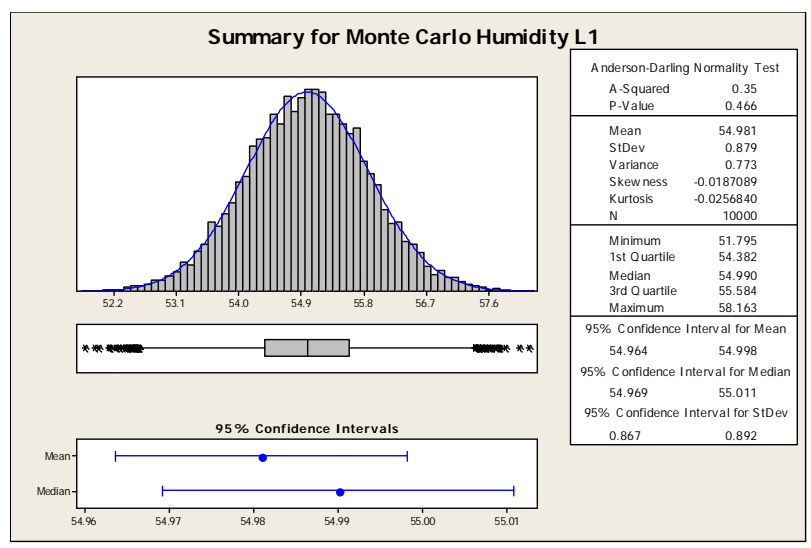

Figure 9: Summary for Monte Carlo Humidity L1 
Table 2: A List of random variable $\mathrm{N}=99$ Humidity in fire models

A list of random variables $N=10 k$ Humidity in fire size models

\begin{tabular}{lllllll}
\hline Variable & Distribution & Mean & Std Deviation & Kurtosis & Skrew ness & Units \\
\hline Humd L0 & Normal & 0.2048 & 0.2306 & 0.0221 & 0.0043 & $\mathrm{c}$ \\
Humd L1 & Normal & 54.981 & 0.879 & -0.01257 & -0.01871 & $\mathrm{c}$ \\
& & & & & & \\
Humd L2 & Normal & 69.269 & 1.758 & -0.016 & -0.0336 & $\mathrm{c}$ \\
Humd L3 & Normal & 71.342 & 1.834 & -0.009 & -0.0252 & $\mathrm{c}$ \\
\hline
\end{tabular}

The distribution Minitab output from the results in figure 9 was used to generate 10,000 random sample of outcome for each control strategies as shown in Table 2.

The dataset output that was generate indicates that the distribution is normally distributed with mean 58.981with a standard deviation of 0.879 . The AD test statistic confirms the results with A-squared value of 0.35 and P-value of $0.466(\mathrm{P}>0.005)$. On average, the smallest value was 51.795 and the largest value was 58.163, thus giving the range of 109.98 .The transmitted variation results in a standard deviation 0.879 , which is the estimated value for standard uncertainly[13].

Both the mean and standard deviations are within the $95 \%$ confident level of estimation, thus giving a probability coverage of $95 \%$ with a low endpoint of 54.964 and a high endpoint of 54.998 for mean. Similarly, the $95 \%$ standard deviation has a low end point of 0.867 and high endpoint of 0.892 .

Box plot diagram is used to show a snapshot picture of overall pattern of disturbance with respect to chasis twisted. The dark overlapping dotted line indicates the extend of disturbance, if a very large sample from normal distribution is generated 10000 samples[14]. The magnitude of disturbance is given by maximum $=58.163$ and minimum 51.795 .

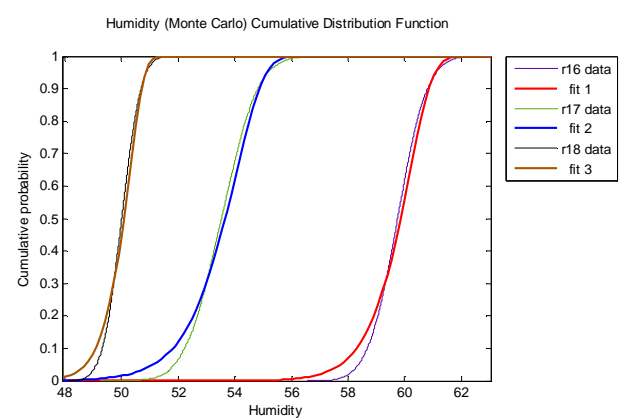

Figure 10: Monte Carlo Cumulative Distribution Function

The distribution of fire sizes and fire growth parameter values observed were both reasonably well approximated by the log-normal distribution.

From CDF diagram of Figure 10: Cumulative Distribution Function finding the Humidity can be read by Matlab simulation. For the $\mathrm{L} 0=51, \mathrm{~L} 1=51<\mathrm{h}<56$ and $\mathrm{L} 2=$
$<56$ readings can be facilitated to design algorithm for variable parameters Humidity.

Algorithm 1: Classification of fire distribution( Humidity)

1. Read humidity value, $\boldsymbol{h}$

2. If $\boldsymbol{h}<\mathbf{5 1}$ then is level (LO)

3. else if $\mathbf{5 1}<\boldsymbol{h}<\mathbf{5 6}$ then is level (L1)

4. else if 56> is level (L2)

5. output display level:

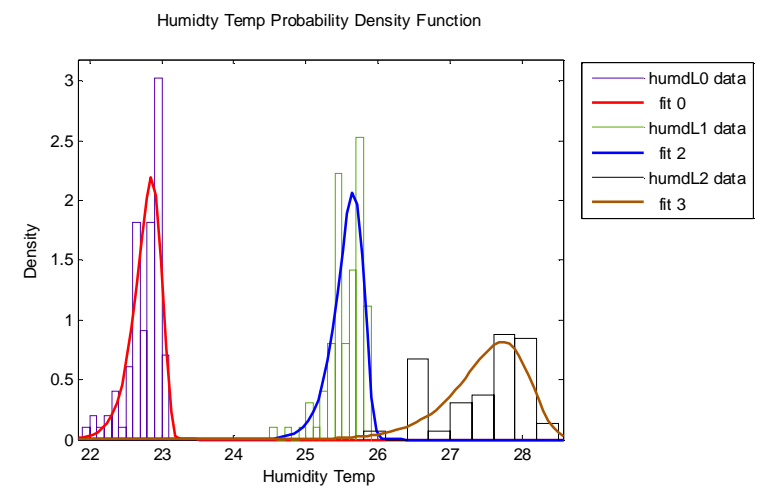

Figure 11: Summary of Monte Carlo of Humidity Temp L1

PDF diagram of Figure 11: Probability Density Function find the Humidity can be read by Matlab simulation. For the L0 = $23, \mathrm{~L} 1=23<\mathrm{h}<26$ and $\mathrm{L} 2=>26$ readings can be facilitated to design algorithm for variable parameters Humidity Temp[14].

Algorithm 2: Classification of fire distribution ( Humidity Temp)

1. Read Hump Temp value, $\boldsymbol{h}$

2. If $\boldsymbol{h}<\mathbf{2 4}$ then is level (LO)

3. else if $\mathbf{2 4}<h<26$ then is level (L1)

4. else if 26> is level (L2)

5. output display level:

\section{CONCLUSION}

This paper presents a stochastics analysis of the compartment fire using graphical minitab simulation. In addition to reliability analysis, the method also allow efficient system analysis using samples conditional on failure. Based on the experiments that have been carried out different types of fires can be made by making a few methods of engineering analysis sthochastic where type of distribution sketched by fire as a small fire, big fire, and from the experiments to determine the risk of a fire that could endanger consumers. Stochastic method successfully distinguishes type of fire. The effects of the experiment we can determine the impact of these risks. If we can measure as little as 1 candle fires, of course, a huge fire-giving on fire risk can be measured and identified. From 4 variable data taken shows two variables such as temperature 
data. Humidity is very significant in this study. Temperature data and pressure distribution function shows a significant effect may be due to others factors, including wind, environment and others. Through this study fires can be avoided and consequently reduce damage caused by fire including loss of life. From the graph plots could easily give the parameters and algorithm development.

\section{REFERENCES}

1. F.Yang, X.Qian, and P. Huang, "Fire Safety AssessmentUnderground Buildings based on Grey Relational Analysis," Procedia Eng., vol. 45, pp. 89-95, 2012.

https://doi.org/10.1016/j.proeng.2012.08.126

2. W. Lee, M. Cheon, C.-H. Hyun, and M. Park, "Development of building fire safety system with automatic security firm monitoring capability," Fire Saf. J., vol. 58, pp. 65-73, May 2013. https://doi.org/10.1016/j.firesaf.2013.01.003

3. C. Ramírez-Marengo, C. Diaz-Ovalle, R. Vázquez-Román, and M. S. Mannan, "A stochastic approach for risk analysis in vapor cloud explosion," J. Loss Prev. Process Ind., vol. 35, pp. 249-256, May 2015.

4. L. Mathelin, M. Y. Hussaini, and T. a. Zang, "Stochastic approaches to uncertainty quantification in CFD simulations," Numer. Algorithms, vol. 38, no. 1-3, pp. 209236, 2005. https://doi.org/10.1007/BF02810624

5. Y. Jin and B.-S. Jang, "Probabilistic fire risk analysis and structural safety assessment of FPSO topside module," Ocean Eng., vol. 104, pp. 725-737, Aug. 2015.

6. S. K. Au, Z.-H. Wang, and S.-M. Lo, "Compartment fire risk analysis by advanced Monte Carlo simulation," Eng. Struct., vol. 29, no. 9, pp. 2381-2390, 2007.

7. A. Camillo, E. Guillaume, T. Rogaume, A. Allard, and F. Didieux, "Risk analysis of fire and evacuation events in the European railway transport network," Fire Saf. J., vol. 60, pp. 25-36, Aug. 2013.

8. H. Frantzich, "Uncertainty and Risk Analysis in Fire Safety Engineering," Dep. Fire Saf. Eng. Lund Inst. Technol., vol. PhD, p. 206, 1998.

9. M. Kobes, I. Helsloot, B. de Vries, and J. G. Post, "Building safety and human behaviour in fire: A literature review," Fire Saf. J., vol. 45, no. 1, pp. 1-11, 2010.

10. H. Sharma and V. K. Sachan, "Online Monitoring Inside a Building Based on Energy Efficient Wireless Sensor Network," vol. 4, no. 2, pp. 1-5.

11. G. Welch and G. Bishop, "An Introduction to the Kalman Filter,” In Pract., vol. 7, no. 1, pp. 1-16, 2006.

12. L. Fang, J. Yang, J. Zu, G. Li, and J. Zhang, "Quantifying influences and relative importance of fire weather, topography, and vegetation on fire size and fire severity in a Chinese boreal forest landscape," For. Ecol. Manage., vol. 356, pp. 2-12, Mar. 2015.

https://doi.org/10.1016/j.foreco.2015.01.011

13. X. Zhang, X. Li, and G. Hadjisophocleous, "A probabilistic occupant evacuation model for fire emergencies using Monte Carlo methods," Fire Saf. J., vol. 58, pp. 15-24, May 2013.
14. S.V.R.K.Rao, M.Saritha Devi, A.R.Kishore and Praveen Kumar "Wireless sensor Network based Industrial Automation using Internet of Things(IoT)," IJATCSE 2018.Vol.7.6

https://doi.org/10.30534/ijatcse/2018/01762018

15. K.Vallimadhavi , P.Sivaprasad; R.Baladinakar, and Y.D.Siva Prasad., "Automatic connection of various medical sensors by using WBAN adaptive routing protocol" IJATCSE 2018.Vol.7.6 https://doi.org/10.30534/ijatcse/2018/14762018

16. Lloret, J.; Tomas, J.; Garcia, M.; Canovas, A. A hybrid stochastic approach for self-location of wireless sensors in indoor environments. Sensors 2009, 9, 3695- 3712.

17. Son, B.; Her, Y.; Kim, J. A design and implementation of forest-fires surveillance system based on wireless sensor networks for South Korea Mountains. IJCSNS 2006, 6, 124-130.12

18.Hefeeda, M.; Bagheri, M. Forest fire modeling and early detection using wireless sensor networks. Ad Hoc Sens.Wirel. Networks 2009, 7, 169-224.

19. Lloret, J.; Tomas, J.; Garcia, M.; Canovas, A. A hybrid stochastic approach for self-location of wireless sensors in indoor environments. Sensors 2009, 9, 3695-3712. 